\title{
Initial Error Growth and Predictability of Chaotic Low-dimensional Atmospheric Model
}

\author{
Hynek Bednář Aleš Raidl Jiři Mikšovský \\ Department of Meteorology and Environment Protection, Faculty of Mathematics and Physics, Charles University in Prague, \\ V Holešovičkách 2, 180 00, Prague 8, Czech Republic
}

\begin{abstract}
The growth of small errors in weather prediction is exponential on average. As an error becomes larger, its growth slows down and then stops with the magnitude of the error saturating at about the average distance between two states chosen randomly. This paper studies the error growth in a low-dimensional atmospheric model before, during and after the initial exponential divergence occurs. We test cubic, quartic and logarithmic hypotheses by ensemble prediction method. Furthermore, the quadratic hypothesis suggested by Lorenz in 1969 is compared with the ensemble prediction method. The study shows that a small error growth is best modeled by the quadratic hypothesis. After the error exceeds about a half of the average value of variables, logarithmic approximation becomes superior. It is also shown that the time length of the exponential growth in the model data is a function of the size of small initial error and the largest Lyapunov exponent. We conclude that the size of the error at the least upper bound (supremum) of time length is equal to 1 and it is invariant to these variables. Predictability, as a time interval, where the model error is growing, is for small initial error, the sum of the least upper bound of time interval of exponential growth and predictability for the size of initial error equal to 1 .
\end{abstract}

Keywords: Chaos, planetary atmospheres, prediction methods, error analysis, modeling.

\section{Introduction}

Due to the fact that the atmosphere is a chaotic dynamical system, the growth of small errors in weather prediction is exponential. In the case of sufficiently small initial error, the governing equations can be linearized, which leads to exponential growth of the error. Generally, whether an error is small enough to guarantee the exponential growth depends on the specific meteorological conditions and/or the model under study. The issue of small errors growth in the context of the model studied in this paper was also addressed in [1-5]. For a more comprehensive introduction to the problem of weather predictability we refer readers to the book of Palmer and Hagedorn ${ }^{[6]}$.

If the system which governs the change of the error is linear, then the exponential growth will continue unabated. The earth's atmosphere is a non-linear system and as the error becomes larger, the growth rate decreases. Eventually, all systematic growth should stop and the size of the error should be equal to the average size of the distance of two randomly chosen states.

Predictability and average initial error growth for numerical weather prediction models (NWPM) are still in the focus of researchers, e.g. [7, 8]. Analysis of the last mentioned growth was firstly performed by Lorenz ${ }^{[9]}$ in 1969 . He introduced a quadratic hypothesis which is based on the assumption that if the principal nonlinear terms in the atmospheric equations are quadratic, then the nonlinear terms in the equations governing the field of errors will also be quadratic but he could not prove this theory, because the

Regular Paper

Special Issue on Recent Advances on Complex Systems Control, Special Issue on Recent
Modelling and Prediction

Manuscript received August 8, 2013; revised October 28, 2013

This work was supported by Research Plan (No. MSM0021620860) and by project (No.SVV-2013-267308). method that has to be adopted (for NWPM we know just model state and we do not know the real state) gives limited number of initial error sizes and limited available data for valid approximation. This problem is still present. In this case, it is a logical step to use a less complex experimental model that gives the possibilities to choose "real" and model states and to choose the number of approximated data. The present study attempts to examine this hypothesis, using the low-dimensional atmospheric model introduced by Lorenz in $1996^{[10]}$.

Even for systems with exponential error growth, it does not start right from the initial time, and we observe the transient behavior instead. After the transient behavior dies out and exponential growth follows, the time length of this part will be governed by the size of small initial error and by the model parameters. Predictability, as a time interval where the model error is growing, is also expected to be a function of the above mentioned errors and parameters. We also investigate this phenomenon.

Some results on the topic were published by us in a more compact form in [1].

\section{Model}

Lorenz $^{[10]}$ introduced a model of nonlinear behavior, with $N$ variables $X_{1}, \cdots, X_{N}$ connected by governing equations:

$$
\frac{\mathrm{d} X_{n}}{\mathrm{~d} t}=-X_{n-2} X_{n-1}+X_{n+1} X_{n-1}-X_{n}+F
$$

where $X_{n-2}, X_{n-1}, X_{n}, X_{n+1}$ are unspecified (i.e., unrelated to actual physical variables) scalar meteorological quantities, $F$ is a constant representing external forcing, and $t$ is time. The index is cyclic so that $X_{n-N}=X_{n+N}=$ $X_{n}$, and the variables can be viewed as existing around a circle. The nonlinear terms of (1) simulate advection. The 
linear terms represent mechanical and thermal dissipation. The model can quantitatively describe the weather system to a certain extent; but instead of the well-known Lorenz's model of atmospheric convection ${ }^{[11]},(1)$ cannot be derived from any atmospheric dynamic equations. The motivation was to formulate the simplest possible set of dissipative chaotically behaving differential equations that share some properties with the "real" atmosphere. In $[2,3]$, the reasoning for usability of such a model was discussed in more detail.

Details of the numerical integration of (1) are given in Appendix. For our computation we choose $N=36$, so each sector covers 10 degrees of longitude. Parameters $F$ were selected equal to 8,9 and 10 , successively. We first choose arbitrary values of the variables, and use a fourth order Runge-Kutta method with a time step $\Delta t=0.05$ or 6 hours, we integrate forward for 14400 steps, or 10 years. We then use the final values, which should be more or less free of transient effect. For individual parameters $F$, we estimate the global largest Lyapunov exponents $\lambda_{\max }$ by the method of numerical calculation presented in [12]. We gradually get

$$
F=(8 ; 9 ; 10) \rightarrow \lambda_{\max }=(0.33 ; 0.39 ; 0.46) .
$$

By the definition in [9], a bounded dynamical system with a positive Lyapunov exponent is chaotic. Because all values of the largest Lyapunov exponents of the model are positive and the system is bounded ${ }^{[2-4]}$, its chaoticity has been established for all three values of $F$. Strictly speaking, we also need to exclude the asymptotically periodic behavior, but such a task is impossible for numerical simulation to fulfill. The choice of parameters $F$ and time unit $=5$ days is made to obtain the same values of the largest Lyapunov exponents as the state of the art models of complete global atmospheric circulation.

\section{$3 \quad$ Ensemble prediction method}

The ensemble prediction method employed is similar to the one in [10] and is used to calculate the average initial error growth. We make an initial "run" by choosing error $e_{n 0}$ and letting $X_{n 0}^{\prime}=X_{n 0}+e_{n 0}$ be the "observed" initial value of $N$ variables. We then integrate forward from the true and the observed initial state, for 50 days ( $K=200$ steps), obtaining $N$ sequences $X_{n 0}, \cdots, X_{n K}$ and $X_{n 0}^{\prime}, \cdots, X_{n K}^{\prime}$. After that, we let $e_{n k}=X_{n k}^{\prime}-X_{n k}$ for all values of $k$ and $n$. To get more representative values, we make a total of $M=250$ runs in the same manner by letting new values of $X_{n 0}$ be the old values of $X_{n K}$ in each run. Finally, we let

$$
e^{2}(\tau)=\frac{1}{N}\left(e_{1 k}^{2}+\cdots+e_{N k}^{2}\right)
$$

be the average of the $N$ values, where $\tau=k \Delta t$ is the predictable range and

$$
\log E^{2}(\tau)=\frac{1}{M}\left(\log e^{2}(\tau)_{1}+\cdots+\log e^{2}(\tau)_{M}\right)
$$

is the average of $M$ values. The logarithmic average is chosen because of its suitability for comparison with growth governed by the largest Lyapunov exponent. For further information, see [13-15].

\section{Quadratic hypothesis}

According to Lorenz ${ }^{[10]}$, there is an eventual cessation of the exponential growth due to the processes represented by nonlinear terms in the weather governing equations. The most important are the quadratic terms, which represent the advection of the temperature and velocity fields. Under the assumption that the principal nonlinear terms in the atmospheric equations are quadratic, the nonlinear terms in the equations governing the field of errors will also be quadratic. To describe this, Lorenz ${ }^{[10]}$ defined

$$
\frac{\mathrm{d} E(t)}{\mathrm{d} t}=a E(t)-b E(t)^{2}
$$

where $E(t)$ is a distance at time $t$ between two originally nearby trajectories; and $a, b$ are constants. The quadratic hypothesis is also used to describe the behavior of initial error growth, for example in [16,17].

\subsection{Method}

Because we want to study behavior of (2), we make differences $y_{k}=\frac{(E(\tau+\Delta t)-E(\tau))}{\Delta t}$ at points $x_{k}=\frac{(E(\tau)+E(\tau+\Delta t))}{2}$, where $E$ is average initial error growth calculated from the ensemble prediction method (Section 3).

Next we interpolate the data $\left(x_{k}, y_{k}\right)$. The interpolation equations are

$$
\begin{aligned}
& y(t)=\frac{\mathrm{d} E(t)}{\mathrm{d} t}=a E(t)-b E(t)^{2} \\
& y(t)=\frac{\mathrm{d} E(t)}{\mathrm{d} t}=a E(t)-b E(t)^{3} \\
& y(t)=\frac{\mathrm{d} E(t)}{\mathrm{d} t}=a E(t)-b E(t)^{4} \\
& y(t)=\frac{\mathrm{d} E(t)}{\mathrm{d} t}=-a E(t) \ln (b E(t)) .
\end{aligned}
$$

Equation (2) represents the examined quadratic hypothesis. The alternative forms (3) and (4) are added, because Lorenz ${ }^{[9]}$ noticed that the cubic and quartic equations would also fit his data. Equation (5) is chosen because if $Q(t)=\ln (\overline{E(t)})$, with $\bar{E}$ being the normalized $E$, then $\frac{\mathrm{d} Q(t)}{\mathrm{d} t}=a\left(1-\mathrm{e}^{Q(t)}\right)$ represents the quadratic hypothesis. In [18], it was assumed that linear fit $\frac{\mathrm{d} Q(t)}{\mathrm{d} t}=-a Q(t)$ is superior to the quadratic hypothesis. Parameters $a$ and $b$ in $(2-5)$ are examined and discussed in the next section.

\subsection{Results}

Different initial errors $e_{0}$ exhibit different behaviors of an error growth. To study that, we selected six magnitudes of $\left\|e_{0}\right\|$ for each $F:\left\|e_{0,1}\right\|=0.0001,\left\|e_{0,2}\right\|=0.001$, $\left\|e_{0,3}\right\|=0.01,\left\|e_{0,4}\right\|=0.1,\left\|e_{0,5}\right\|=0.6,\left\|e_{0,6}\right\|=1$, where $\|\cdot\|$ marks the Euclidean norm. The interpolation equations were tested for all three values of parameter $F$ and initial error $e_{0}$. Table 1 shows the RMS error between values obtained from the ensemble prediction and from the interpolation equations. The error is divided by the average value of $\frac{(E(\tau+\Delta t)-E(\tau))}{\Delta t}$. Fig. 1 displays the error growth rate $\frac{\mathrm{d} E}{\mathrm{~d} t}$ versus $E$ for all parameters $F$ and for $e_{0,2}, e_{0,4}$ and $e_{0,5}$. Each interpolation equation gives specific values of $a$ and $b$ for particular $F$ and $e_{0}$. Our aim is to find a general 
Table 1 RMS error between values obtained from the ensemble prediction and from the interpolation equations. The error is divided by the average value of $\frac{(E(\tau+\Delta t)-E(\tau))}{\Delta t}$ and displayed in percent. Gray cells mark values with the best results

\begin{tabular}{|c|c|c|c|c|c|c|c|c|c|c|c|c|c|c|c|c|c|c|c|}
\hline & \multirow{3}{*}{$\begin{array}{c}\text { Initial error } \\
\qquad F\end{array}$} & \multicolumn{18}{|c|}{ Percent error } \\
\hline & & \multicolumn{3}{|c|}{0.0001} & \multicolumn{3}{|c|}{0.001} & \multicolumn{3}{|c|}{0.01} & \multicolumn{3}{|c|}{0.1} & \multicolumn{3}{|c|}{0.6} & \multicolumn{3}{|c|}{1} \\
\hline \multirow{5}{*}{$\begin{array}{c}\text { Interpolation } \\
\text { equation }\end{array}$} & & 8 & 9 & 10 & 8 & 9 & 10 & 8 & 9 & 10 & 8 & 9 & 10 & 8 & 9 & 10 & 8 & 9 & 10 \\
\hline & $a x-b x^{2}$ & 16 & 19 & 12 & 13 & 11 & 13 & 16 & 12 & 14 & 16 & 15 & 14 & 34 & 27 & 29 & 47 & 41 & 36 \\
\hline & $a x-b x^{3}$ & 23 & 27 & 22 & 23 & 22 & 20 & 21 & 22 & 25 & 29 & 25 & 27 & 40 & 38 & 38 & 53 & 50 & 51 \\
\hline & $a x-b x^{4}$ & 30 & 29 & 32 & 30 & 29 & 28 & 28 & 33 & 32 & 37 & 32 & 35 & 48 & 44 & 43 & 60 & 52 & 55 \\
\hline & $a x \ln (b x)$ & 20 & 21 & 19 & 22 & 23 & 21 & 22 & 22 & 22 & 22 & 25 & 21 & 26 & 21 & 23 & 39 & 31 & 34 \\
\hline
\end{tabular}

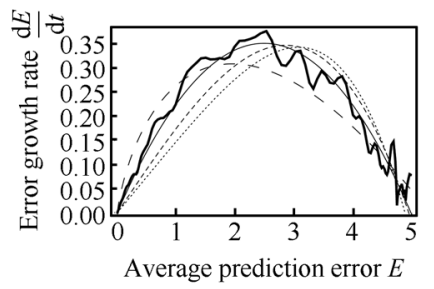

(a) $F=8, e_{02}=0.001$

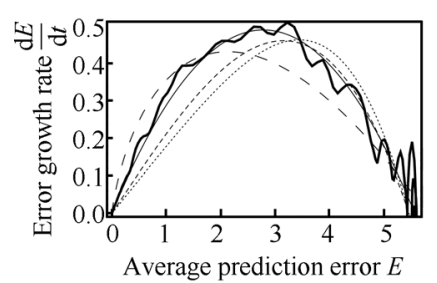

(d) $F=9, e_{02}=0.001$

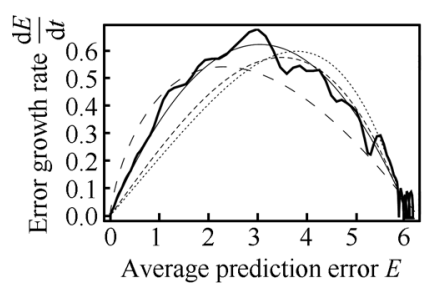

(g) $F=10, e_{02}=0.001$

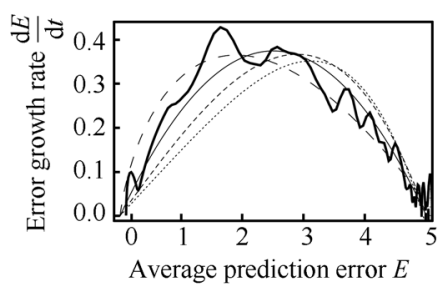

(b) $F=8, e_{04}=0.1$

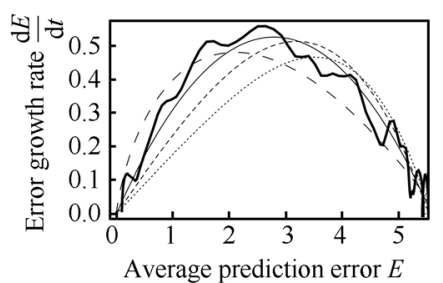

(e) $F=9, e_{04}=0.1$

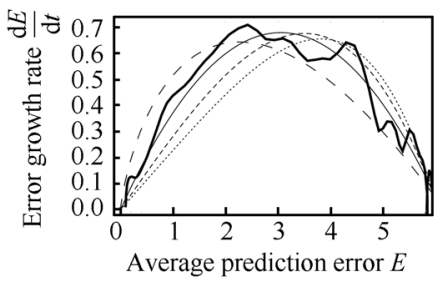

(h) $F=10, e_{04}=0.1$

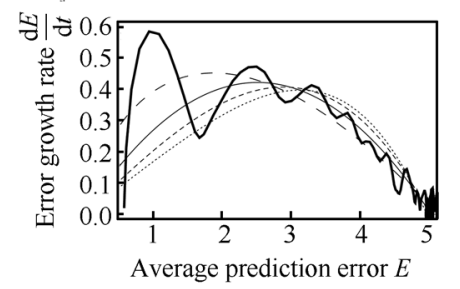

(c) $F=8, e_{05}=0.6$

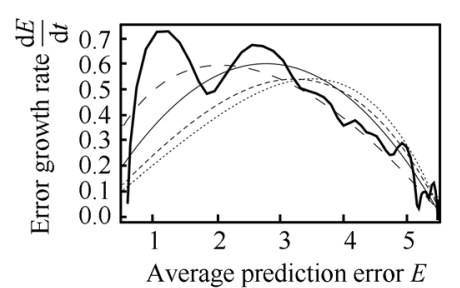

(f) $F=9, e_{05}=0.6$

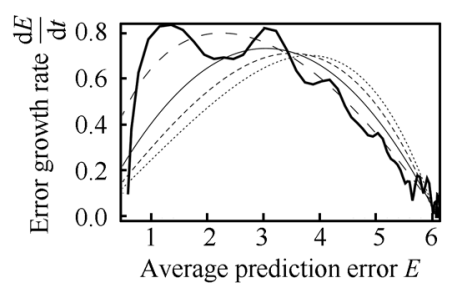

(i) $F=10, e_{05}=0.6$

Fig. 1 The error growth rate $\frac{\mathrm{d} E}{\mathrm{~d} t}$ versus $E$ for all parameters of $F$ and for $e_{0,2}, e_{0,4}$ and $e_{0,5}$. The thick line represents ensemble prediction, the thin line corresponds to (2), the dashed line to (3), the thinly dashed line to (4) and the largely dashed line to (5)

description of $a$ and $b$ by well-known parameters of the system. The early growth rate should be close to $\frac{\mathrm{d} E}{\mathrm{~d} t}=\lambda_{\max } E$. That means $a=\lambda_{\max }$ for all interpolation equations. Results for $(2-4)$ are the following. The constant $a$ measures the growth rate of small errors, the quadratic (cubic, quartic) term has to be negative if $a$ is positive, since it is the only factor that can stop the growth. If $E$ is normalized such that the value which it approaches as $t \rightarrow \infty$ is unity, $b=a$. For unnormalized $E, b=\frac{\lambda_{\max }}{E^{*}}$ for $(2), b=\frac{\lambda_{\max }}{E^{* 2}}$ for equation (3) and $b=\frac{\lambda_{\max }}{E^{* 3}}$ for (4), where $E^{*}$ denotes the saturation value for $E$. For (5), then $b=\frac{1}{E^{*}}$.

From Table 1 and Fig. 1, it is obvious that the most accurate and therefore usable hypotheses are the quadratic (2) and logarithmic (5) ones. The theoretical values of parameters $a$ and $b$ would make the inaccuracy of cubic and quartic hypotheses even greater. Therefore, we will from now on work only with quadratic and logarithmic hypotheses. Table 2 shows the RMS error between values obtained from the ensemble prediction and from $(2-5)$, where parameters $a$ and $b$ are the expected theoretical values. We can see a higher increase of the percent error for (5) than for (2). The difference between Table 2 and Table 1 is displayed in Table 3.

\section{Exponential growth and predictabil- ity}

Usability of the exponential model of initial error growth is depended on the size of initial error as well as the model parameter $F$. In the introduction, we mentioned that the exponential growth $E_{\exp }(t)=e_{0} \mathrm{e}^{\left(\lambda_{\max } t\right)}$ governed by the largest Lyapunov exponent $\lambda_{\max }$ occurs in the case of a sufficiently small initial error $e_{0}$. In this section, we present sizes of this initial error and therefore the usability of the exponential model.

Predictability $t_{p}$ is the time interval where systematic growth of initial error occurs and the size of this error is 
smaller than the average size of the distance of two randomly chosen states. This section also focuses on the dependence of $t_{p}$ on the size of initial error, parameter $F$ and on the possible connection between predictability and exponential growth.

\subsection{Method}

If we display the time variation of the average prediction error $E$ obtained from the Lorenz's model and exponential growth (Fig. 2), we can hardly decide whether the expo- nential growth happens or not. We could get same guesses for predictable time read from graphs of time evolution of $E$ (Fig. 2), but we would rather relate it to a better specified value instead of the saturated value $E^{*}$. In both cases we want to get more precise values and accuracy, therefore we have to introduce a more sophisticated method. This method was developed from the assumption that if the exponential growth is present, then the ratio of the two average errors $E$ calculated from ensemble prediction method (Section 3) in two following time steps $\Delta t$ is

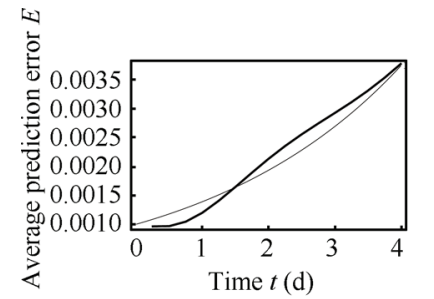

(a) $F=8, e_{02}=0.001, t \in(0 ; 4)$

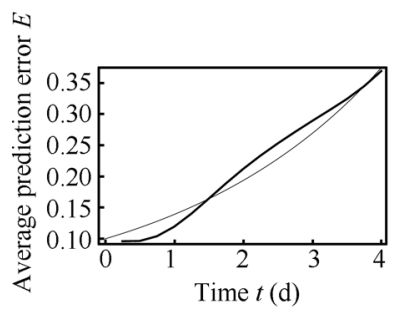

(d) $F=8, e_{04}=0.1, t \in(0 ; 4)$

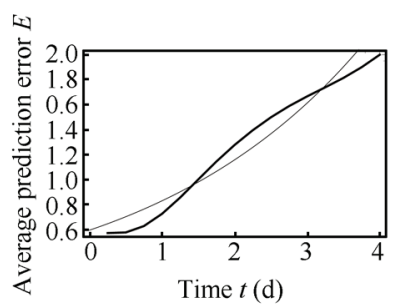

(g) $F=8, e_{05}=0.6, t \in(0 ; 4)$

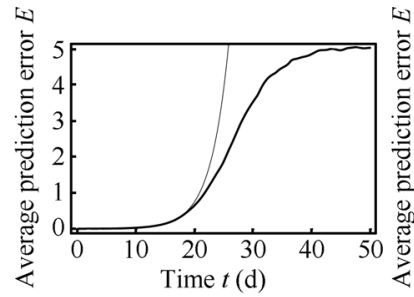

(b) $F=8, e_{02}=0.001, t \in(0 ; 50)$

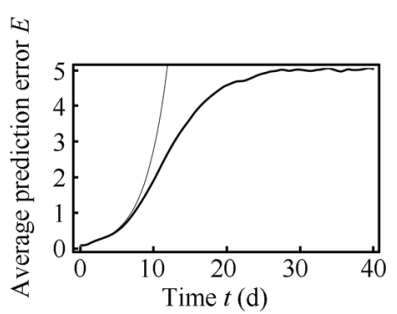

(e) $F=8, e_{04}=0.1, t \in(0 ; 40)$

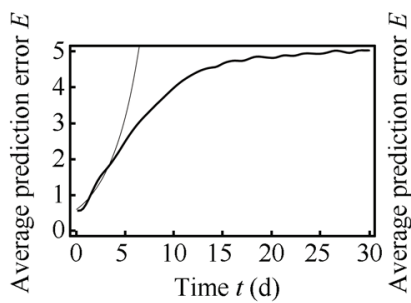

(h) $F=8, e_{05}=0.6, t \in(0 ; 30)$

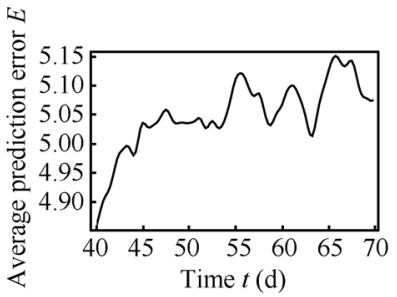

(c) $F=8, e_{02}=0.001, t \in(0 ; 70)$

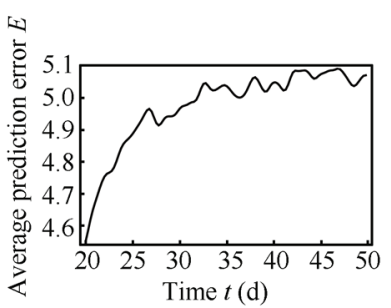

(f) $F=8, e_{04}=0.1, t \in(20 ; 50)$

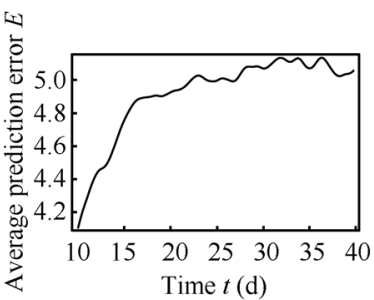

(i) $F=8, e_{05}=0.6, t \in(10 ; 40)$

Fig. 2 Time variations of the average prediction error $E$ obtained from the Lorenz's model (the thick line) for $F=8$, for $e_{0,2}, e_{0,4}$, $e_{0,5}$ and exponential growth governed by the largest Lyapunov exponent (the thin line)

Table 2 The RMS error between values obtained from the ensemble prediction and from $(2,5)$, where parameters $a$ and $b$ are the expected theoretical values. The error is divided by the average value of $\frac{(E(\tau+\Delta t)-E(\tau))}{\Delta t}$ and displayed in percent

\begin{tabular}{|c|c|c|c|c|c|c|c|c|c|c|c|c|c|c|c|c|c|c|c|}
\hline & & \multicolumn{18}{|c|}{ Percent error } \\
\hline & \multirow{2}{*}{$\begin{array}{c}\text { Initial error } \\
F\end{array}$} & \multicolumn{3}{|c|}{0.1} & \multicolumn{3}{|c|}{0.1} & \multicolumn{3}{|c|}{0.1} & \multicolumn{3}{|c|}{0.1} & \multicolumn{3}{|c|}{0.6} & \multicolumn{3}{|c|}{1} \\
\hline & & 8 & 9 & 10 & 8 & 9 & 10 & 8 & 9 & 10 & 8 & 9 & 10 & 8 & 9 & 10 & 8 & 9 & 10 \\
\hline Interpolation & $a x-b x^{2}$ & 32 & 25 & 29 & 30 & 20 & 19 & 22 & 20 & 16 & 18 & 13 & 15 & 34 & 29 & 28 & 48 & 45 & 40 \\
\hline equation & $a x \ln (b x)$ & 97 & 89 & 94 & 83 & 85 & 82 & 73 & 77 & 84 & 73 & 63 & 72 & 42 & 41 & 46 & 39 & 36 & 34 \\
\hline
\end{tabular}

Table 3 The absolute values of the differences between Table 2 and Table 1

\begin{tabular}{|c|c|c|c|c|c|c|c|c|c|c|c|c|c|c|c|c|c|c|c|}
\hline & \multirow{3}{*}{$\begin{array}{c}\text { Initial error } \\
F\end{array}$} & \multicolumn{18}{|c|}{ Percent error } \\
\hline & & \multicolumn{3}{|c|}{0.0001} & \multicolumn{3}{|c|}{0.001} & \multicolumn{3}{|c|}{0.01} & \multicolumn{3}{|c|}{0.1} & \multicolumn{3}{|c|}{0.6} & \multicolumn{3}{|c|}{1} \\
\hline & & 8 & 9 & 10 & 8 & 9 & 10 & 8 & 9 & 10 & 8 & 9 & 10 & 8 & 9 & 10 & 8 & 9 & 10 \\
\hline Interpolation & $a x-b x^{2}$ & 16 & 6 & 17 & 17 & 9 & 6 & 6 & 8 & 2 & 2 & 2 & 1 & 0 & 2 & 1 & 1 & 4 & 4 \\
\hline equation & $a x \ln (b x)$ & 67 & 68 & 75 & 61 & 62 & 61 & 51 & 55 & 62 & 51 & 38 & 51 & 16 & 20 & 23 & 0 & 5 & 0 \\
\hline
\end{tabular}




$$
\frac{E(\tau)}{E(\tau-\Delta t)}=\frac{E_{\exp }(\tau)}{E_{\exp }(\tau-\Delta t)}=\mathrm{e}^{\left(\lambda_{\max } \Delta t\right)}
$$

and hence it follows that

$$
G(\tau)=\frac{\ln \left(\frac{E(\tau)}{E(\tau-\Delta t)}\right)}{\left(\lambda_{\max } \Delta t\right)}=1 .
$$

Boundaries of predictability occur when

$$
E(\tau)=E(\tau+\Delta t)=E^{*}
$$

which means that $G(\tau)=0$. Through the use of $G$, we analyze exponential growth and predictability.

\subsection{Results}

Function $G$ is calculated for a variety of initial errors $e_{0}$ and parameters $F$. We again choose six magnitudes of $\left\|e_{0}\right\|$ for each parameter $F$ : $\left\|e_{0,1}\right\|=0.0001,\left\|e_{0,2}\right\|=$ $0.001,\left\|e_{0,3}\right\|=0.01,\left\|e_{0,4}\right\|=0.1,\left\|e_{0,5}\right\|=0.2,\left\|e_{0,6}\right\|=1$, where $\|\cdot\|$ marks the Euclidean norm. In Table 4, the time interval $t_{e}$, during which results from ensemble prediction method are close to the theoretical exponential growth, is displayed for each initial error $e_{0}$ and each parameter of $F$. In the same table, predictability $t_{p}$ (length of time interval where $E$ is growing) is also displayed. Fig. 3 shows time evolution of function $G$ for all $F$ and $e_{0,2}, e_{0,4}, e_{0,5}$. The experimental data oscillate around theoretically expected values, and therefore intervals $t_{e}$ and $t_{p}$ are measured as the length with similar oscillation around theoretically expected values rather than an exact match. Table 4 and Fig. 3 also illustrate that the exponential growth is present for $e_{0}$ which is smaller than $e_{0,4}=0.1$ and never starts at the beginning of the growth. There is always a wave that precedes it (it can also be seen in Table 3) and its length is similar throughout the spectrum of $F$ and $e_{0}$. To analyze this behavior further, we focus on the dependence of the least upper bound (supremum) $t_{e, u}$ of time interval $t_{e}$ and predictability $t_{p}$ on natural logarithm of initial errors from $e_{0,1}$ to $e_{0,4}$ for all parameters of $F$ (Fig. 4) and on natural logarithm of initial errors from $e_{0,1}$ to $e_{0,6}$ (Fig. 5) in the second case.

The results in Figs. 4 and 5 indicate the linear dependence between $t_{e, u}$ as well as between $t_{p}$ and $\ln \left(e_{0}\right)$. To get more information, we linearly interpolated the experimental data. The interpolation equations $t_{e, u}\left(e_{0}\right)$ and $t_{p}\left(e_{0}\right)$ for all parameters $F$ were

$$
t_{e, u}=c+d \ln \left(e_{0}\right)
$$

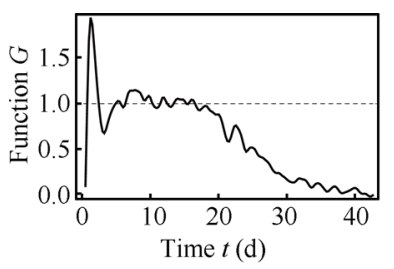

(a) $F=8, e_{02}=0.001$

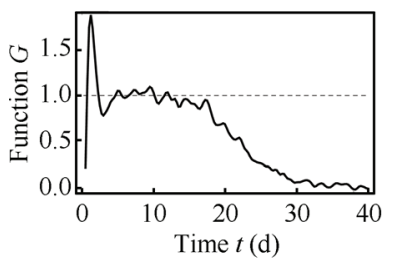

(d) $F=9, e_{02}=0.001$

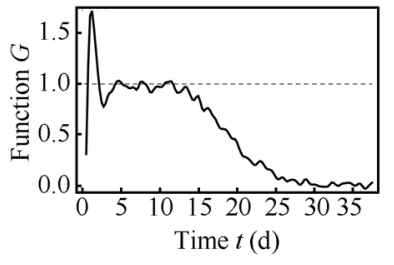

(g) $F=10, e_{02}=0.001$

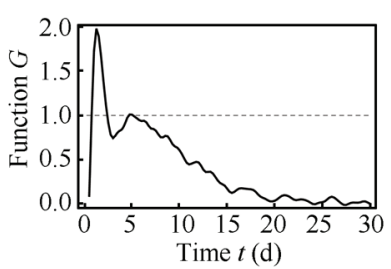

(b) $F=8, e_{04}=0.1$

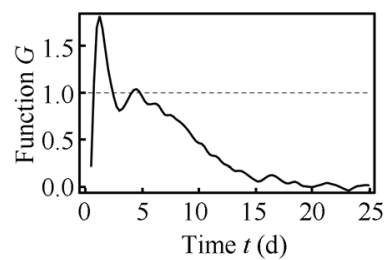

(e) $F=9, e_{04}=0.1$

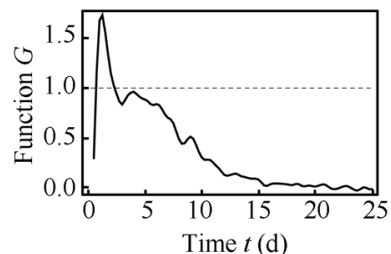

(h) $F=10, e_{04}=0.1$

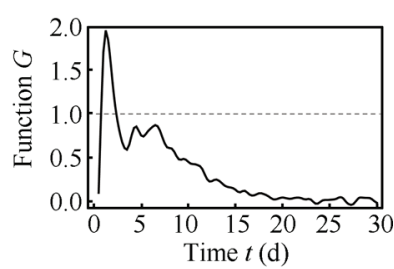

(c) $F=8, e_{05}=0.2$

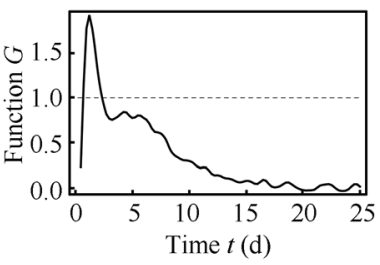

(f) $F=9, e_{05}=0.2$

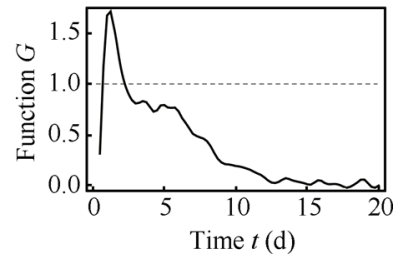

(i) $F=10, e_{05}=0.2$

\begin{tabular}{|c|c|c|c|c|c|c|c|c|c|c|c|c|c|c|c|c|c|c|}
\hline$e_{0}$ & & 0.0001 & & & 0.001 & & & 0.01 & & & 0.1 & & & 0.2 & & & 1 & \\
\hline$F$ & 8 & 9 & 10 & 8 & 9 & 10 & 8 & 9 & 10 & 8 & 9 & 10 & 8 & 9 & 10 & 8 & 9 & 10 \\
\hline$t_{e}(\mathrm{~d})$ & $(6 ; 27)$ & $(5 ; 23)$ & $(4 ; 20)$ & $(5 ; 21)$ & $(5 ; 18)$ & $(4 ; 15)$ & $(5 ; 14)$ & $(4 ; 11)$ & $(4 ; 10)$ & 6 & 5 & 4 & $\mathrm{~N}$ & $\mathrm{~N}$ & $\mathrm{~N}$ & $\mathrm{~N}$ & $\mathrm{~N}$ & $\mathrm{~N}$ \\
\hline$t_{p}(\mathrm{~d})$ & 49 & 40 & 32 & 42 & 34 & 27 & 35 & 27 & 22 & 28 & 21 & 17 & 25 & 19 & 15 & 20 & 15 & 12 \\
\hline
\end{tabular}

Fig. 3 Time evolution of function $G(\tau)=\frac{\ln \left(\frac{E(\tau)}{E(\tau-\Delta t)}\right)}{\lambda_{\max } \Delta t}$ for all parameters of $F$ and for $e_{0,2}, e_{0,4}$ and $e_{0,5}$

Table 4 Time interval $t_{e}$, where results from the ensemble prediction method are close to theoretical exponential growth (N means negative result) and predictability $t_{p}$ (time intervals, where $E$ is growing) for each initial error $e_{0}$ and each parameter $F$ 


$$
\begin{gathered}
t_{p}=f+h \ln \left(e_{0}\right) \\
\left(c_{F=8} ; c_{F=9} ; c_{F=10}\right)=(0.2 ;-0.2 ;-0.2) \\
\left(d_{F=8} ; d_{F=9} ; d_{F=10}\right)=(-3 ;-2.6 ;-2.2) \\
\left(f_{F=8} ; f_{F=9} ; f_{F=10}\right)=(20 ; 15 ; 12) \\
\left(h_{F=8} ; h_{F=9} ; h_{F=10}\right)=(-3.1 ;-2.7 ;-2.2) .
\end{gathered}
$$

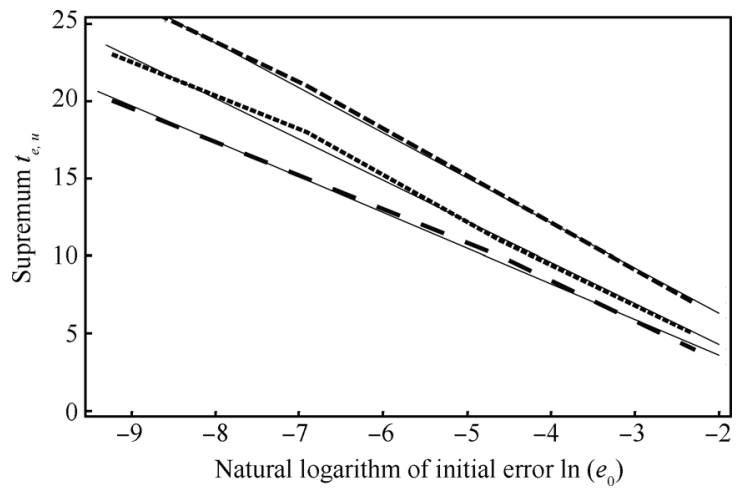

Fig. 4 Time length $t_{e}$ versus natural logarithm of initial errors from $e_{0,1}$ to $e_{0,4}$ for all parameters of $F$. The dashed line is for $F=8$, the thin dashed line is for $F=9$, the large dashed line is for $F=10$ and solid lines represent linear interpolations

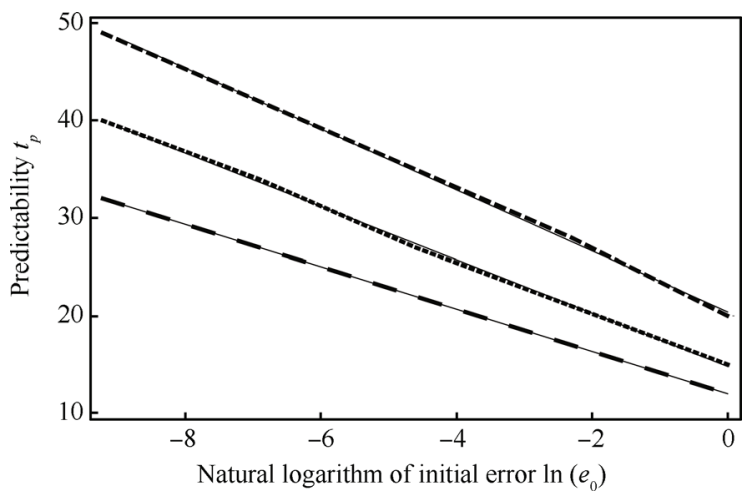

Fig. 5 Time length $t_{p}$ versus natural logarithm of initial errors from $e_{0,1}$ to $e_{0,6}$ for all parameters of $F$. The dashed line is for $F=8$, the thin dashed line is for $F=9$, the large dashed line is for $F=10$ and solid lines represent linear interpolations

Equations (6) and (7) suggest that as we choose a bigger initial error, the window of exponential growth and predictability decreases with the natural logarithm of the initial error. Coefficient $c$ is close to 0 for all $F$. Coefficient $f$ is the same as $t_{p}$ of $e_{0,6}$ for all $F$. Coefficients $d$ and $h$ are similar to each other. Theoretically, possible values for the coefficients come from the definition of exponential growth

$$
E_{\exp }(t)=e_{0} \mathrm{e}^{\left(\lambda_{\max } t\right)}
$$

thus

$$
t\left(e_{0}\right)=-\frac{1}{\lambda_{\max }} \ln \left(\frac{e_{0}}{E_{\exp }}\right) .
$$

Values of $\frac{1}{\lambda_{\max }}$ are

$$
\left(\frac{1}{\lambda_{\max , F=8}} ; \frac{1}{\lambda_{\max , F=9}} ; \frac{1}{\lambda_{\max , F=10}}\right)=(3.03 ; 2.56 ; 2.17) \text {. }
$$

If we compare it with $d$ and $h$, we see similarity of the results.

From (6) and (7), it is obvious that function $t_{p}\left(t_{e, u}\right)$ (Fig. 6) is linear:

$$
\begin{gathered}
t_{p}=o+p t_{e, u} \\
\left(o_{F=8} ; o_{F=9} ; o_{F=10}\right)=(20 ; 15 ; 12) \\
a\left(p_{F=8} ; p_{F=9} ; p_{F=10}\right)=(1 ; 1 ; 1) .
\end{gathered}
$$

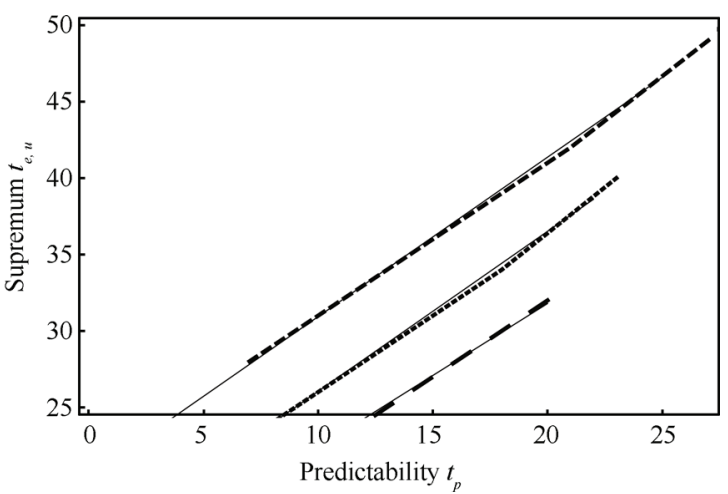

Fig. 6 Predictability $t_{p}$ versus time length $t_{e, u}$. The dashed line is for $F=8$, the thin dashed line is for $F=9$, the large dashed line is for $F=10$ and solid lines are linear interpolations

If the exponential growth is present, $t_{p}$ is equal to $t_{e, u}$ plus $e_{0,6}$ for all parameters of $F$.

\section{Discussion}

The Lorenz's results ${ }^{[9]}$ fulfilled the cubic relation (3) fairly well, though he only used limited number of data for his study. We showed that neither (3) nor (4) fits our data properly, compared to the other alternatives. Two usable hypotheses approximating the error growth rate are

$$
\begin{gathered}
\frac{\mathrm{d} E(t)}{\mathrm{d} t}=\lambda_{\max } \cdot E(t)-\left(\frac{\lambda_{\max }}{E^{*}}\right) \cdot E(t)^{2} \\
\frac{\mathrm{d} E(t)}{\mathrm{d} t}=-\lambda_{\max } \cdot E(t) \cdot \ln \left(\frac{E(t)}{E^{*}}\right)
\end{gathered}
$$

where $\lambda_{\max }$ is the largest Lyapunov exponent and $E^{*}$ is the saturated value of $E$.

If we look for the best approximation of model values of the error growth rate $\dot{E}$, then the quadratic law (2) fits the best for $e_{0}$ up to about 0.1. For higher values, it is better to use logarithmic law (5). On the other hand, if we want to estimate parameters of the model or use $(9,10)$ directly, it is, according to Fig. 2, Table 2 and Table 3, better to use (9) for $e_{0}=\langle 0 ; 1\rangle$ and (10) for $e_{0}=\langle 1 ; 2\rangle$. The reason for the difference can be found in Fig. 7 . We can estimate that the best result for (2) is the range of $e_{0}$ between 0.001 and 0.1 and for (5) we obtain the range of $e_{0}$ between 1 and 1.5. The difference between theoretical and experimental data for parameter $a$ is much higher for (5) than for (2) and parameter $b$ in (5) is inside the logarithmic function and therefore any possible difference would be increased by this function. 




(a) Parameter $a$, (2)

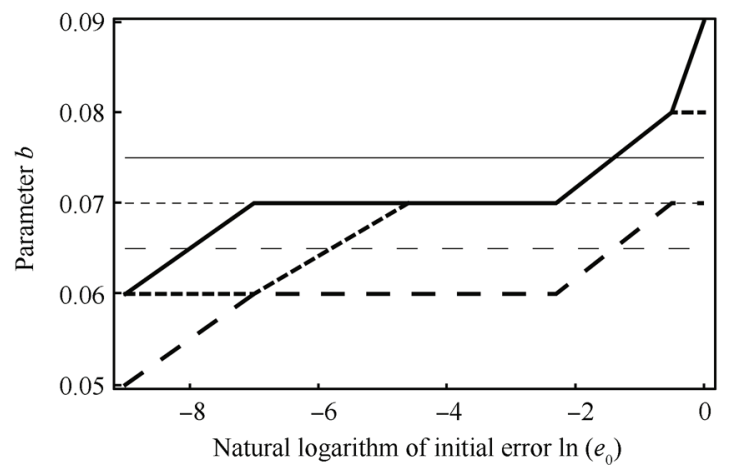

(b) Parameter $b,(2)$

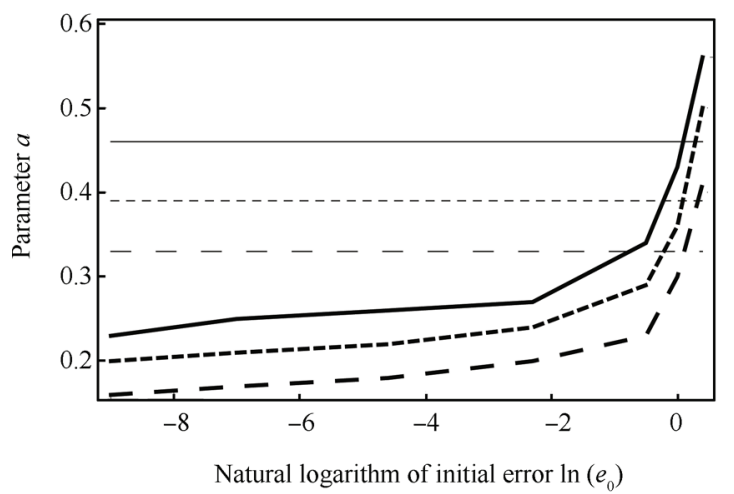

(c) Parameter $a,(5)$

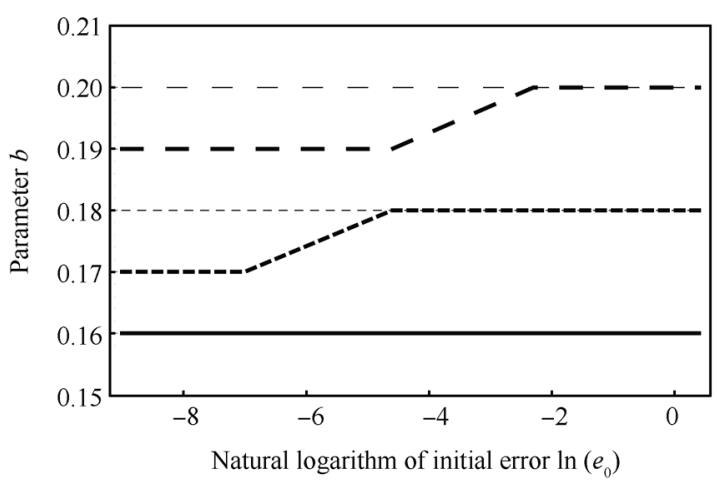

(d) Parameter $b,(5)$

Fig. 7 Comparison of theoretical and experimental parameters $a, b$ for $(2,5)$. The thin dashed lines represent $F=8$, the dashed lines are for $F=9$ and the solid lines are for $F=10$. The thin lines are theoretical values and the thick lines are experimental values
Here, we also need to mention that variables $X_{1}, \cdots, X_{N}$ vary between approximately -6 and +12 . Fig. 8 shows time variations of $X_{1}$ during a period of 180 days. The average value $\bar{X}$ of $X_{n}$ is 2 , which means that for $e_{0} \leqslant \frac{\bar{X}}{2}$, it is better to use (9) and for $e_{0} \geqslant \frac{\bar{X}}{2}$ it is better to use logarithmic law (10). This is in good agreement with [18], where the same was suggested.

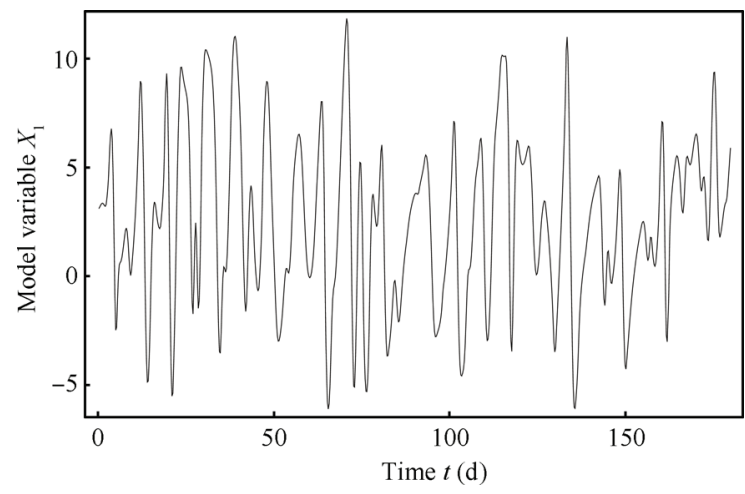

Fig. 8 Time variations of $X_{1}$ during a period of 180 days

Solutions of (9) and (10) are

$$
\begin{aligned}
& E(t)=\frac{E^{*}}{\left(\frac{E^{*}}{e_{0}}-1\right) \mathrm{e}^{\left(-\lambda_{\max } t\right)}+1} \\
& E(t)=E^{*}\left(\frac{e_{0}}{E^{*}}\right)^{\mathrm{e}^{\left(-\lambda_{\max } t\right)}} .
\end{aligned}
$$

The maximum sufficiently small initial error with the exponential growth of $E(t)$ is $e_{0}=0.1$ (according to Table 4 and Fig. 3). If we take a look at the validity of quadratic hypothesis with experimental parameters, we can see the same maximal value. We can speculate that it is not a coincidence that a sufficiently small error is directly connected with the quadratic hypothesis, and that if we use a sufficiently small initial error, then the growth will be governed by (9).

The greatest lower bound (infimum) $t_{e, l}$ of the time interval $t_{e}$ has a similar value across the spectrum of $e_{0}$ (Table 4 ), and we did not find any interpolation equation. The behavior of $E(t)$ from the ensemble prediction approach before the exponential growth takes place has a similar form for all $F$ and $e_{0}$ (Table 4, Figs. 2 and 3). Errors measured by ensemble prediction approach are approximately decreasing during the first 0.3 days and after 0.5 days it is approximately as large as $e_{0}$. Exponential growth overestimates the error for approximately the first 1.3 days, while underestimates it (till $t_{e, l}$ ) later. The maximum of this wave behavior occurs approximately at 2.3 days. Same behavior was also observed for $e_{0} \geqslant 0.1$. To explain it we have to remind the definition of Lyapunov exponent as a longterm average characteristic. As stated in $[19,20]$, the error growth differs from the one established from Lyapunov exponent for the first few days. That is true not only for low dimensional models, but also for more complex global atmospheric circulation models.

The least upper bound $t_{e, u}$ of time interval $t_{e}$ follows (6), 


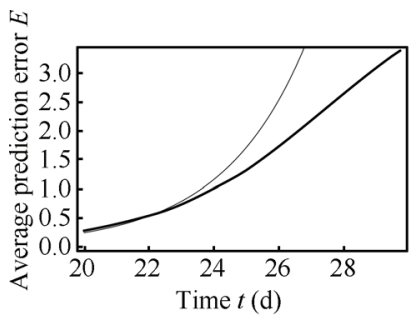

(a) $F=9, e_{01}=0.0001, t \in(20 ; 30)$

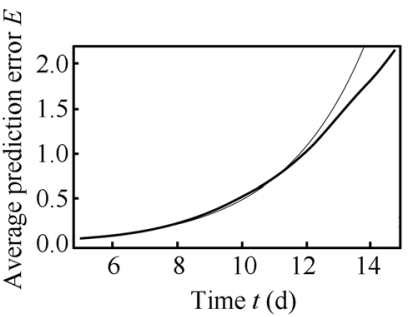

(b) $F=9, e_{03}=0.01, t \in(5 ; 15)$

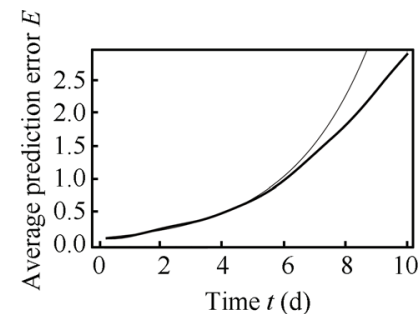

(c) $F=9, e_{n 4}=0.1, t \in(0 ; 10)$

Fig. 9 Time variations of the average prediction error $E$ around $E=1$ obtained from the Lorenz's model (the thick line) for $F=9$, for $e_{0,1}, e_{0,3}, e_{0,4}$ and exponential growth governed by the largest Lyapunov exponent (the thin line)

which can be generally written as

$$
t_{e, u, \text { theoretical }}=-\left(\frac{1}{\lambda_{\max }}\right) \ln \left(e_{0}\right) .
$$

If we substitute (3) into the equation for the exponential growth $E_{\exp , u}(t)=e_{0} \mathrm{e}^{\left(\lambda_{\max } t\right)}$, we get

$$
E_{e, u, \text { theoretical }}=1 \text {. }
$$

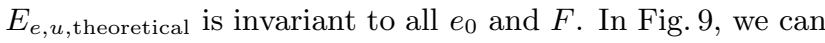
see that $E_{e, u}$ from the ensemble prediction approach is very similar.

Here we would like to remind the reader that it is better to use (9) for $e_{0}=\langle 0 ; 1\rangle$ and (10) for $e_{0}=\langle 1 ; 2\rangle$, and again we dare to say that it is not a coincidence that the value of 1 plays a role in both. Equation (7) approximating predictability $t_{p}$ (length of the time interval, where $E$ is growing) with theoretical parameters $f$ and $h$ can be written as

$$
t_{p}=t_{p, e_{0}=1}-\left(\frac{1}{\lambda_{\max }}\right) \ln \left(e_{0}\right)
$$

where $t_{p, e_{0}=1}$ is the predictability for $e_{0}=1$. We did not find any general expression for $t_{p, e_{0}=1}$. The second part of

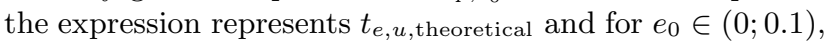
(15) can be rewritten into

$$
t_{p}=t_{p, e_{0}=1}+t_{e, u, \text { theoretical }}
$$

which is the same equation as (8). This means that for $e_{0} \in(0 ; 0.1)$ the increase in predictability time $t_{p}$ is due to the increase in time length of the exponential growth $t_{e, u}$. The maximum predictability governed by (11) is 49 days for $F=8$ and $e_{0,1}$. The lower predictability, governed by (12), is 12 days for $F=10$ and $e_{0,6}$ (Table 4 ).

\section{Conclusion}

This article focuses on analyzing the average error growth in a low-dimensional atmospheric model ${ }^{[10]}$. Theoretical hypotheses with experimental and theoretical coefficients and exponential model are compared with the ensemble prediction method for different initial errors and model parameters. Dependence of predictability and validity of exponential growth on lastly mentioned errors and parameters is also studied.
The important resulting values are 0.1 and 1 . Value 0.1 is border for hypotheses with experimental coefficients, and it is also the maximum sufficiently small initial error with exponential growth. If the initial error is smaller than 0.1 , then it is better to use quadratic hypothesis. If it is bigger, then the logarithmic hypothesis becomes superior. Value 1 is border for hypotheses with theoretical coefficients, and it is also the size of the error at least upper bound (supremum) of time length of exponential growth for all sufficiently small initial errors and model parameters. If the initial error is smaller than 1, then it is better to use quadratic hypothesis. If it is bigger, then the logarithmic hypothesis becomes superior. Predictability, as a time interval where the model error is growing, is, for small initial error, the sum of the least upper bound of time interval of exponential growth and the predictability for the size of initial error equal to 1 as shown in (16). The least upper bound of time interval of exponential growth is negatively proportional to Lyapunov exponent and directly proportional to natural logarithm of small initial error as shown in (15). Exponential growth does not start from the beginning of error growth and the greatest lower bound of time interval of exponential growth has similar values across the spectrum of sufficiently small initial errors.

It is in relatively good agreement, e.g., $[7,8]$, with the ability and predictability of the current meteorological models (the predictability is approximately two weeks).

This number (two weeks) itself cannot be transferred to dynamic systems of a different nature (nonlinear oscillators, mechanical systems, etc.). When studying the predictability of other systems it is possible to use the methodology of our article, for example, a combination of approach using Lyapunov exponents and ensemble prediction or estimation error growth depending on its stage.

\section{Appendix}

Numerical integration of (1) is performed by RungeKutta method of 4th order, as described by many authors, e.g. [21-23]. The algorithm is implemented in software Wolfram Mathematica ${ }^{\mathrm{TM}}$. This integration scheme has been studied in terms of stability and usability for chaotic systems in [24]. The study shows that the use of integration step 0.05 is quite sufficient. Moreover, we have verified using different integration parameters that the cited method provides a sufficiently smooth trajectory in phase space of the system under study. The same method and time step were also used for solving equation (1) in $[2,6,10]$. 


\section{References}

[1] H. Bednar A. Raidl, J. Mikšovský. Initial errors growth in chaotic low-dimensional weather prediction model. In Proceedings of Nostradamus International Conference on Prediction, Modeling and Analysis of Complex Systems, Springer, Nostradamus, Ostrava, Czech Republic, vol. 210, pp. 333-342, 2013.

[2] E. N. Lorenz, K. A. Emanuel. Optimal sites for supplementary weather observations: Simulation with a small model. Journal of the Atmospheric Sciences, vol. 55, no. 3, pp. 399414, 1998.

[3] E. N. Lorenz. Designing chaotic models. Journal of the Atmospheric Sciences, vol. 62, no. 5, pp. 1574-1587, 2005.

[4] S. Herrera, D. Pazó, J. Fernández, M. A. Rodríguez. The role of large-scale spatial patterns in the chaotic amplification of perturbations in a Lorenz' 96 model. Tellus Series A - Dynamic Meteorology and Oceanography, vol.63, no. 5, pp. 978-990, 2011.

[5] H. M. Arnold, I. M. Moroz, T. N. Palmer. Stochastic parametrizations and model uncertainty in the Lorenz' 96 system. Philosophical Transactions of the Royal Society A - Mathematical Physical and Engineering Sciences, vol. 371, no. 1991, 20110179, 2013.

[6] T. Palmer, R. Hagedorm. Predictability of Weather and Climate, Cambridge, UK: Cambridge University Press, pp. 1$702,2006$.

[7] L. S. R. Froude, L. Bengtsson, K. I. Hodges. Atmospheric predictability revisited. Tellus Series A - Dynamic Meteorology and Oceanography, vol. 65, pp. 1-13, 2013.

[8] L. Magnusson, E. Kallen. Factors influencing skill improvements in the ECMWF forecasting system. Monthly Weather Review, vol. 141, no. 9, pp. 3142-3153, 2013.

[9] E. N. Lorenz. Atmospheric predictability as revealed by naturally occurring analogs. Journal of the Atmospheric Sciences, vol. 26, no. 4, pp. 636-646, 1969.

[10] E. N. Lorenz. Predictability: A problem partly solved. In Proceedings of Seminar on Predictability, CMWF, Reading, Berkshire, UK, vol. 1, pp. 1-18, 1996.

[11] E. N. Lorenz. Deterministic nonperiodic flow. Journal of the Atmospheric Sciences, vol. 20, no. 2, pp. 130-141, 1963.

[12] J. C. Sprott. Chaos and Time-Series Analysis, New York, USA: Oxford University Press, pp. 1-507, 2003.

[13] A. Trevisan. Impact of transient error growth on global average predictability measures. Journal of the Atmospheric Sciences, vol. 50 no. 7, pp. 1016-1028, 1993.

[14] R. Q. Ding, J. P. Li. Comparisons of two ensemble mean methods in measuring the average error growth and the predictability. Acta Meteorologica Sinica, vol. 25, no. 4, pp. 395-404, 2011.

[15] R. Benzi, F. C. Carnevale. A possible measure of local predictability. Journal of the Atmospheric Sciences, vol. 46, no. 23, pp. 3595-3598, 1989.

[16] E. N. Lorenz. Atmospheric predictability experiments with a large numerical model. Tellus, vol. 34, no. 6, pp. 505-513, 1982.

[17] L. Bengtsson, K. I. Hodges. A note on atmospheric predictability. Tellus, vol. 58, no. 1, pp. 154-157, 2006.

[18] A. Trevisan, P. Malguzzi, M. Fantini. On Lorenz's law for the growth of large and small errors in the atmosphere. Journal of the Atmospheric Sciences, vol. 49 no. 8, pp. 713$719,1992$.
[19] J. Smagorinsky. Problems and promises of deterministic extended range forecasting. Bulletin of the American Meteorological Society, vol. 50, no. 5, pp. 286-311, 1969.

[20] E. N. Lorenz. Deterministic nonperiodic flow. Journal of the Atmospheric Sciences, vol. 20, no. 2, pp. 130-148, 1963.

[21] E. W. Weisstein. Runge-Kutta method, [Online], Available: http: //mathworld. wolfram. com/Runge-Kutta Method.ht$\mathrm{ml}$, September 11, 2013.

[22] J. D. Lambert, D. Lambert. Numerical Methods for Ordinary Differential Systems: The Initial Value Problem, New York, USA: Wiley, pp. 149-205, 1991.

[23] J. H. E. Cartwright, O. Piro. The dynamics of RungeKutta methods. Bifurcations Chaos, vol. 2, no. 3, pp. 427449, 1992.

[24] H. Bednář Ensemble Experiments with Low-dimensional Atmospheric Models, Ph. D. dissertation, Charles University in Prague, Czech Republic, 2010.

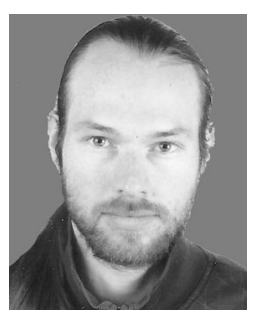

Hynek Bednár̆ received his B.Sc. degree in general physics and M. Sc. degree in meteorology and climatology from the Faculty of Mathematics and Physics, Charles University in Prague, Czech Republic in 2008 and 2010, respectively. Currently he is a Ph. D. candidate in Department of Meteorology and Environment Protection at the same university, working on his dissertation Earth Atmosphere". entitled "Study of Chaotic Behavior of the

His research interests include nonlinearity and predictability of the atmosphere, initial error growth of atmospheric models and low-dimensional models.

E-mail: hynek.bednar@mff.cuni.cz (Corresponding author)

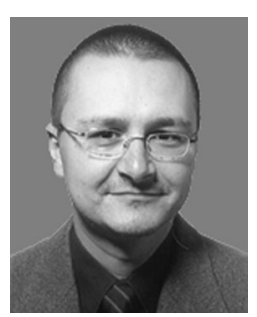

Aleš Raidl received his M. Sc. degree in physics and $\mathrm{Ph}$. D. degree in meteorology and climatology from the Faculty of Mathematics and Physics, Charles University in Prague, Czech Republic in 1992 and 1996, respectively. Now he is a lecturer at the Department of Meteorology and Environment Protection, Charles University, Czech Republic. He is the co-author of three monographs on deterministic chaos and strange kinetics with applications in physics, and nonlinear problems of geophysical hydrodynamics.

His research interests include issues of nonlinear geophysical hydrodynamics and problems of deterministic chaos and predictability in atmospheric models. He also focuses on the area of nonlinear analysis and modeling of experimental data.

E-mail: ales.raidl@mff.cuni.cz

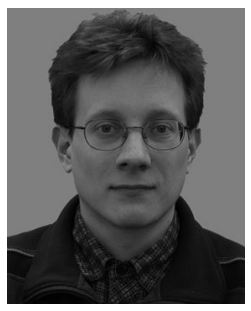

Jiří Mikšovský received his M. Sc. degree in physics and $\mathrm{Ph} . \mathrm{D}$. degree in meteorology and climatology from the Faculty of Mathematics and Physics, Charles University in Prague, Czech Republic in 2000 and 2004, respectively. Now he is a senior assistant professor at Charles University in Prague.

His research interests include time series search. and data analysis in the field of climate re- 